\title{
Traqueomalacia pediátrica
}

\author{
Pediatric tracheomalacia
}

Andrés Alvo V1,2,3, Cecilia Sedano $\mathbf{M}^{1}$, Graeme van der Meer ${ }^{3}$.

\begin{abstract}
RESUMEN
La malacia de la vía aérea central puede afectar la tráquea y/o los bronquios, haciéndola susceptible al colapso de sus paredes durante el ciclo respiratorio. Puede clasificarse como primaria o secundaria y clínicamente se manifiesta por síntomas respiratorios recurrentes o persistentes (especialmente en espiración), infecciones recurrentes y en casos severos, episodios de hipoventilación con cianosis. El diagnóstico se establece mediante broncoscopía flexible o rígida. Los estudios de imágenes se consideran complementarios, siendo especialmente útiles en casos de duda diagnóstica, estudio de causas secundarias o para la planificación preoperatoria. Su tratamiento depende de distintos factores como la severidad de los síntomas, su etiología, ubicación y extensión. En casos leves, una observación activa y tratamiento médico en espera de la resolución espontánea en los primeros 2 años, suelen ser suficientes. En casos moderados, la ventilación a presión positiva podría ser de utilidad. En casos severos está indicado el tratamiento quirúrgico, mediante distintas técnicas que buscan dar soporte a la estructura traqueobronquial debilitada, incluyendo traqueostomía, suspensiones directas (traqueopexias) o indirectas (aortopexia), tutores externos o stents intraluminales. En este artículo se revisarán las principales causas y tratamientos disponibles para la traqueomalacia pediátrica. Ya que su diagnóstico y manejo son complejos, es fundamental el trabajo de equipos médicos multidisciplinarios familiarizados con esta patología.
\end{abstract}

Palabras clave: Traqueomalacia, vía aérea pediátrica, estridor, broncoscopía.

\begin{abstract}
Central airway malacia can affect the trachea and/or the main bronchi, making their walls susceptible to collapse during the respiratory cycle. It can be classified as primary or secondary, and clinically presents with recurrent or persistent respiratory symptoms
\end{abstract}

\footnotetext{
1 Departamento de Otorrinolaringología, Universidad de Chile. Santiago, Chile.

2 Unidad de Otorrinolaringología, Hospital Roberto del Río, Santiago, Chile.

3 Department of Paediatric Otorhinolaryngology-Head and Neck Surgery, Starship Children's Hospital, Auckland, Nueva Zelanda.
}

Los autores declaran no tener conflictos de interés.

Recibido el 6 de diciembre, 2018. Aceptado el 20 de enero, 2019. 
(especially on expiration), recurrent infections and in severe cases, episodes of hypoventilation with cyanosis. The diagnosis is established by flexible or rigid bronchoscopy; imaging studies are considered as complementary, especially in cases of unclear diagnosis, secondary causes or for preoperative planning. Treatment depends on different factors such as the severity of the symptoms, their etiology, location and extension. In mild cases, active observation and medical treatment waiting for spontaneous resolution may be enough, which usually occurs in the first 2 years of age. In moderate cases, positive pressure ventilation could be useful. In severe cases, surgical treatment is indicated. Different techniques aiming to provide support to the weakened tracheobronchial structures are available, including tracheostomy, direct (tracheopexies) or indirect suspensions (aortopexy), external splints and intraluminal stents. In this article we present the main etiologies and available treatments for pediatric tracheomalacia. Given that diagnosis and management of these patients is complex, the work of multidisciplinary teams familiar with this pathology is of paramount importance.

Key words: Tracheomalacia, pediatric airway, stridor, bronchoscopy.

\section{INTRODUCCIÓN}

A modo general, podemos definir la traqueomalacia (TM) como una debilidad de las paredes traqueales, que la hacen más susceptible al colapso dinámico ante los cambios de presión. Si esta debilidad compromete también a uno 0 ambos bronquios principales, se utiliza el término tráqueo-broncomalacia (TBM) ${ }^{1}$. La broncomalacia (BM) también puede existir de manera aislada, aunque es mucho menos frecuente. Esta debilidad puede deberse a la atrofia de las fibras elásticas de la pars membranacea en la pared traqueal posterior, a alteraciones de los cartílagos, 0 a ambas $^{2}$. Es habitual la apariencia de un músculo traqueal elongado (Figura 1), aplanamiento anteroposterior de la tráquea y la pérdida de la forma de herradura de los cartílagos traqueales ${ }^{3}$, lo que lleva a la pérdida de la relación normal de 4,5:1 entre cartílago y músculo. A pesar de que se desconoce su real incidencia, se estima que podría ser más frecuente que lo comúnmente pensado. El estudio más citado, realizado en los Países Bajos, estimó que los casos primarios de TM ocurrirían en al menos 1: 2.100 niños, siendo más común en varones ${ }^{4,5}$. El objetivo de este artículo es revisar las principales causas y tratamientos disponibles para la TM pediátrica.

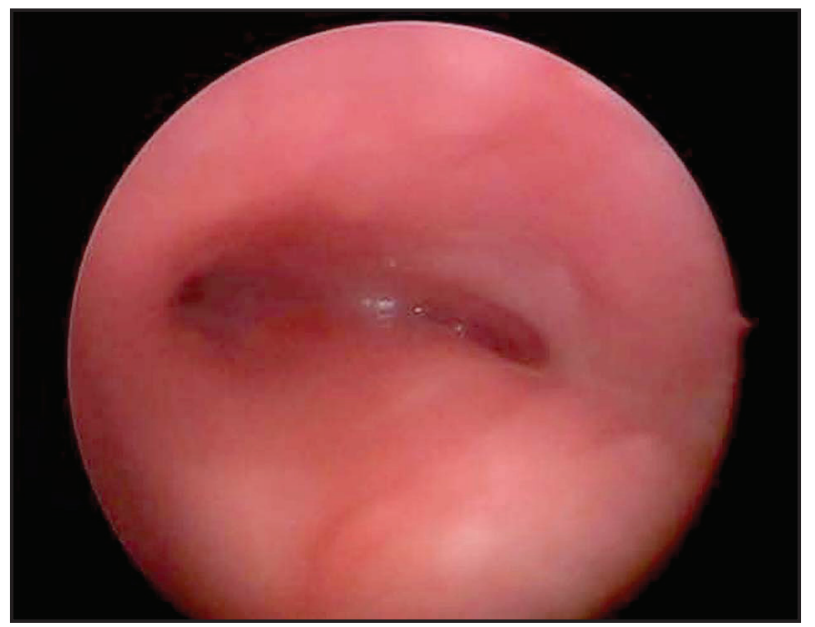

Figura 1. Aspecto endoscópico de una traqueo-broncomalacia primaria. Se puede observar un aplanamiento anteroposterior de la tráquea distal, carina y bronquio principal derecho. La pared posterior traqueal se encuentra ensanchada (músculo traqueal ancho) y los cartílagos han perdido su forma de herradura. 


\section{DEFINICIÓN Y CLASIFICACIONES}

En la actualidad no existe una definición ni clasificación globalmente aceptada de TM. Algunos autores distinguen el término TM (debilidad del cartílago) de la disquinesia traqueal (disfunción de la pared posterior membranosa), mientras que otros lo usan indistintamente ${ }^{6}$. Tampoco se ha establecido el grado de colapso traqueal que debe ser considerado anormal; aunque en general se utiliza un valor arbitrario de $>50 \%$, se sabe que los valores de colapso entre voluntarios sanos y pacientes con TM se sobreponen, al menos en adultos ${ }^{5}$. La TM pediátrica puede dividirse en primaria y secundaria, de acuerdo a si el problema es intrínseco a la tráquea o si existe una alteración subyacente que genere o favorezca la malacia (Tabla 1$)^{6}$. Es aconsejable también distinguir si corresponde a una condición aislada 0 si está asociada a un síndrome. De acuerdo a su extensión, la TM puede ser difusa (más asociada a TM primaria) o localizada (habitualmente en TM secundarias). Algunos autores consideran

Tabla 1. Causas de traqueo(bronco)malacia pediátrica ( ${ }^{*}$ adaptado de referencias 2, 4 y 6)

\begin{tabular}{|c|c|}
\hline \multicolumn{2}{|l|}{ PRIMARIA O CONGÉNITA } \\
\hline Condición & Ejemplos y Comentarios \\
\hline Idiopática & - Presente en infantes sanos, sin otros factores de riesgo \\
\hline Prematurez & $\begin{array}{l}\text { - Habitualmente compromiso difuso } \\
\text { - Puede haber un componente "secundario", relacionado a la ventilación con presión positiva } \\
\text { - Tiende a la recuperación, pero puede demorar meses/años }\end{array}$ \\
\hline Colapso pulsátil sin alteración vascular & - Arteria innominada normal \\
\hline Anormalidades cartilaginosas congénitas & $\begin{array}{l}\text { - Discondroplasia / condromalacia / condrodisplasia } \\
\text { - Policondritis } \\
\text { - Síndrome de Ehlers-Danlos }\end{array}$ \\
\hline $\begin{array}{l}\text { Síndromes congénitos asociados } \\
\text { a TM/TBM }\end{array}$ & $\begin{array}{l}\text { - CHARGE, VACTERL, Trisomía 9, Trisomía 21, DiGeorge, Larsen, Pierre-Robin, etc. } \\
\text { - Mucopolisacaridosis: tráquea de Iongitud normal en un cuerpo pequeño, resultando en } \\
\text { acodamientos y torsiones de la tráquea } \\
\text { - El grado y extensión de la malacia es variable, incluso entre pacientes con el mismo síndrome }\end{array}$ \\
\hline Atresia esofágica y/0 & - Con o sin hendidura laríngea \\
\hline Fístula traqueoesofágica & - Considerada como secundaria por algunos autores \\
\hline \multicolumn{2}{|l|}{ SECUNDARIA O ADQUIRIDA } \\
\hline $\begin{array}{l}\text { Intubación prolongada o traqueostomía } \\
\text { Posquirúrgico } \\
\text { Patología inflamatoria/infecciosa }\end{array}$ & $\begin{array}{l}\text { - Asociado a daño por presión, ventilación con presión positiva, etc. } \\
\text { - Reparación de fístula traqueoesofágica, traqueoplastía por deslizamiento, traqueostomía, etc. } \\
\text { - Traqueobronquitis severa } \\
\text { - } \text { Síndrome de Stevens-Johnson } \\
\text { - } \text { Abscesos } \\
\text { - } \text { Cuerpos extraños } \\
\text { - Ingestión de baterías } \\
\text { - Daño traqueobronquial traumático }\end{array}$ \\
\hline & \\
\hline \multicolumn{2}{|l|}{ Compresión extrínseca } \\
\hline $\begin{array}{l}\text { b) Cardiaca } \\
\text { c) Tumores y quistes } \\
\text { d) Esquelética }\end{array}$ & $\begin{array}{l}\text { - Arteria innominada aberrante, doble arco aórtico, sling de la arteria pulmonar, arco aórtico } \\
\text { derecho, arteria subclavia derecha aberrante, etc. } \\
\text { - La malacia puede persistir a pesar de la corrección quirúrgica de la compresión vascular } \\
\text { - Hipertrofia auricular izquierda } \\
\text { - Tumores benignos y malignos de cuello y mediastino } \\
\text { - Quistes congénitos } \\
\text { - Linfadenopatías } \\
\text { - Metástasis } \\
\text { - Escoliosis } \\
\text { - Pectus excavatum }\end{array}$ \\
\hline
\end{tabular}


5 zonas: tráquea proximal, media y distal; y bronquios derecho e izquierdo $0^{7,8}$.

\section{CONDICIONES CARACTERÍSTICAMENTE ASOCIADAS A TRAQUEOMALACIA}

\section{Malacia asociada a atresia esofágica y fístula traqueoesofágica}

La TM es un hallazgo frecuente en estos pacientes, pudiendo comprometer la tráquea de manera localizada 0 difusa. Existen diferentes opiniones respecto a si se trataría de una malacia primaria 0 secundaria. Aunque algunos autores refieren la asociación de TM con atresia esofágica en hasta $60 \%$, un estudio reciente describió la presencia de TM en $89,2 \%$. El 60,7\% presentaron síntomas respiratorios persistentes, ALTE (apparent life-threatening events) en $13,3 \%$ y muerte en $3,8 \%$. El $22 \%$ requirió corrección quirúrgica de la TM. Adicionalmente, la presencia de otras malformaciones laringotraqueales alcanzó $27,2 \%$. Los casos leves podrían manejarse de manera conservadora, pero los severos habitualmente requieren cirugía (frecuentemente una aorto-pexia) asociada 0 no a cirugía para el reflujo gastroesofágico, en especial si se presenta con apneas en relación a este ${ }^{10}$.

\section{Malacia secundaria a compresiones vasculares}

Constituyen un capítulo especial dentro de las TM. Cada malformación se asocia a hallazgos endoscópicos más o menos característicos, con una compresión pulsátil (a veces sutil) en partes específicas del árbol traqueobronquial11. En algunos casos, la malacia puede persistir incluso después de la corrección quirúrgica de los vasos afectados. Estas anomalías pueden dividirse en anillos completos e incompletos, según si la tráquea queda completamente atrapada 0 si existe una compresión no circunferencial sobre ésta.

\section{Anillos completos}

Son menos frecuentes y tienden a presentarse más precozmente que los incompletos, con síntomas más severos. Generalmente requieren la división quirúrgica del componente menos desarrollado del anillo vascular ${ }^{10}$. Al rodear tanto a la tráquea como al esófago, pueden dar síntomas compresivos de ambos sistemas. El doble arco aórtico es el anillo vascular completo más frecuentemente asociado a TM, y se genera por la persistencia anormal de los 2 cuartos arcos aórticos y ambas aortas dorsales ${ }^{12}$. En la broncoscopía se puede observar una compresión anterior y posterior del lado derecho de la tráquea distal (Figura 2) ${ }^{11-13}$. Existen otros anillos completos, habitualmente en relación a un arco aórtico derecho asociado a otras malformaciones vasculares que completan el anillo.

\section{Anillos incompletos y compresiones vasculares no-circunferenciales}

Aunque existen distintas malformaciones capaces de comprimir y generar malacia de la vía aérea, las más comunes o relevantes son:

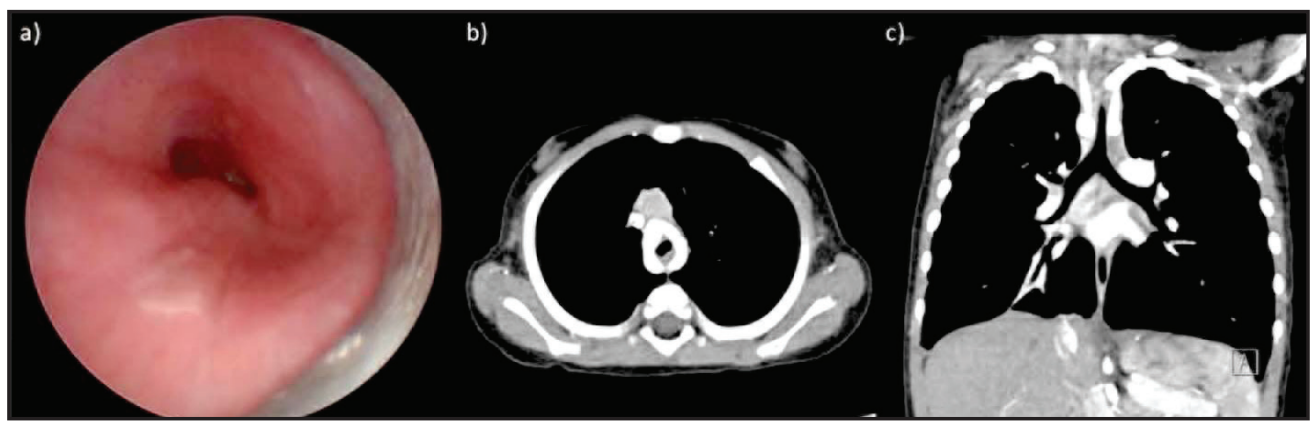

Figura 2. Traqueomalacia en doble arco aórtico. a) Tráqueo-broncoscopía mostrando la imagen característica de compresión anterior y posterior a derecha en la tráquea distal. b) TC de tórax con contraste (corte axial) mostrando el doble arco aórtico rodeando la tráquea. c) TC de tórax con contraste (corte coronal) mostrando los arcos aórticos a ambos lados de la tráquea. 
- Arteria innominada 0 tronco braquiocefálico aberrante, que nace de la aorta en una posición más posteriory a la izquierda, cruzando por delante de la tráquea de manera oblicua ${ }^{10,14}$. Algunos la consideran una entidad sobre diagnosticada y sobre tratada, pues en muchos casos, el origen anatómico de la arteria es normal y el mecanismo principal parece ser una TM primaria. Pese a esto, algunos casos podrían beneficiarse de procedimientos quirúrgicos como arteriopexia 0 reimplantación ${ }^{13}$. La broncoscopía muestra una compresión pulsátil de la pared traqueal anterior, a 1-2 cm de la carina, que al ser comprimida con el broncoscopio contra el esternón genera una disminución del pulso radial derecho $0^{10,14}$.

- Arteria pulmonar izquierda aberrante (sling de la arteria pulmonar), que nace desde el aspecto posterior de la arteria pulmonar derecha, pasando entre la parte distal de la tráquea y el esófago para llegar al hilio del pulmón izquierdo. Genera una compresión del lado derecho de la tráquea distal y del bronquio fuente derecho. Se asocia a estenosis traqueal con anillos traqueales completos en más del $50 \%$ de los casos. Tiene indicación quirúrgica desde su diagnóstico, con reimplantación de la arteria pulmonar izquierda. Si existe una estenosis traqueal asociada, se corrige en la misma cirugía ${ }^{15}$.

\section{DIAGNÓSTICO}

\section{a) Clínica}

En la mayoría de los pacientes, los síntomas aparecen dentro del primer año de vida (el $60 \%$ en los primeros 3 meses). Inicialmente, estos pueden no ser evidentes debido a la menor actividad y requerimientos respiratorios del recién nacido. En la TM primaria, Ios síntomas generalmente tienden a la resolución alrededor de los 2 años, aunque el pronóstico depende de cada $\operatorname{cas}^{10}$. Debido a que la mayoría de las TM comprometen la tráquea intratorácica, el colapso traqueal empeora en espiración cuando la presión intrapleural supera la presión intraluminal. La TM extratorácica es más infrecuente y compromete la tráquea proximal; en ésta, el colapso es predominantemente inspiratorio, cuando la presión intraluminal se hace negativa respecto a la atmosférica ${ }^{16}$.
Las manifestaciones clínicas incluyen tos perruna y tipo "lavadora" (asociado al movimiento de secreciones dentro de la tráquea), estridor, sibilancias, infecciones respiratorias bajas recurrentes, disnea y mala tolerancia al ejercicio; por lo que algunos pacientes son inicialmente diagnosticados como asmáticos. En casos más severos se observan retracciones, episodios de cianosis ("dying spells") e incluso muerte súbita. Los eventos apneicos pueden terminar en apnea refleja y paro respiratorio, con una mortalidad de hasta $80 \%{ }^{1,17,18}$. Otro hallazgo descrito ocasionalmente es la hiperextensión espontánea del cuello, que permitiría tensar y rigidizar la tráquea malácica.

Los pacientes con TM pueden presentar también dificultades para alimentarse (especialmente en casos secundarios a compresión vascular), con disfagia, regurgitación, tos y cianosis. La compresión traqueal por un esófago con contenido y la presencia de reflujo gastroesofágico podrían empeorar los síntomas. A largo plazo, la TM puede llevar también a retención de secreciones y desarrollo de infecciones crónicas al interferir con el aclaramiento mucociliar, alterar el patrón de la tos y por la irritación de las paredes traqueales al contactar entre ellas².

\section{b) Comorbilidades y diagnósticos diferenciales}

Las condiciones asociadas a la TM, tanto primaria como secundaria, incluyen anormalidades cardiovasculares en $20 \%-58 \%$, displasia broncopulmonar en hasta $52 \%$, enfermedad por reflujo gastroesofágico en cerca del $50 \%$ (y hasta $78 \%$ de los casos severos) y otras alteraciones laringotraqueales en porcentajes variables. También se ha descrito una asociación con alteraciones neurológicas en $8 \%-48 \%$ y con retraso severo del desarrollo en $26 \%{ }^{17}$. El diagnóstico diferencial incluye básicamente todas las causas de estridor y sibilancias recurrentes 0 crónicas, incluyendo asma de difícil manejo, obstrucción intraluminal de la vía aérea y aspiración de cuerpos extraños, patologías laríngeas congénitas, entre otras.

\section{c) Endoscopía}

La laringotraqueobroncoscopía se considera el gold standard diagnóstico de la TBM, al permitir 
la visualización directa del grado de colapso, su extensión y las paredes comprometidas. Requiere que el paciente esté en ventilación espontánea, pues si se encuentra paralizado, bajo sedación profunda 0 recibiendo ventilación con presión positiva, el colapso traqueal puede no ser observado $0^{1,16}$. Debe evitarse la hiperextensión del cuello, ya que puede tensar la tráquea, subestimando el grado de colapso.

Los broncoscopios rígidos son los más utilizados debido a su mejor calidad de imagen, posibilidad de conexión al circuito de anestesia y la amplia disponibilidad de instrumentos. Una desventaja es que puede generar distorsión de la vía aérea, lo que puede evitarse introduciendo el broncoscopio sólo hasta la subglotis para ventilar y asegurar la vía aérea, y luego pasar un endoscopio más largo y delgado a través de éste para examinar la vía aérea distal.

Por otra parte, el uso de endoscopios flexibles permite una mejor evaluación dinámica (incluso en pacientes despiertos), genera menos distorsión mecánica de la vía aérea y permite alcanzar segmentos más distales 0 de difícil acceso del árbol traqueobronquial. Sus desventajas son que proporcionan una peor calidad de imagen, la menor gama de instrumentos y que no pueden ser utilizados para ventilar al paciente. Debido a lo anterior, ambas debieran considerarse como técnicas complementarias.

\section{d) Imágenes}

Aún se consideran exámenes de segunda línea en TM primaria, para casos en los que la broncoscopía no puede ser realizada o no permite una evaluación distal a la obstrucción, o ante duda diagnóstica. Por otra parte, las imágenes si tienen un rol importante en el estudio de TM secundarias, en especial para las causas vasculares, y para la planificación preoperatoria de TM con indicación quirúrgica ${ }^{8,10}$.

La radiografía de tórax puede considerarse dentro del estudio inicial, incluso antes de la broncoscopía, para evaluar patologías del parénquima pulmonar y tener una apreciación general de la anatomía del paciente con dificultad respiratoria ${ }^{5}$. Algunos centros aún utilizan estudios esofágicos contrastados, que permiten observar indentaciones esofágicas en casos de anomalías vasculares, además de detectar algunos casos de reflujo gastroesofágico, aspiración y fístulas traqueoesofágicas. El ecocardiograma se utiliza más en el contexto de causas vasculares 0 cardiacas ${ }^{8}$.

El estudio por imágenes de elección en TM es la tomografía computarizada (TC), la cual ha ido adquiriendo un rol cada vez más importante gracias a la aparición de equipos con múltiples detectores (TCMD). Idealmente, las imágenes deben ser obtenidas en inspiración y espiración ${ }^{19}$. En un estudio realizado en pacientes con atresia esofágica y TBM, la TCMD demostró sensibilidades de $67 \%$ para la tráquea superior, $93 \%$ para la tráquea media, 85\% para la tráquea inferior, 100\% para el bronquio principal derecho y $50 \%$ para el izquierdo; con una alta especificidad. Las ventajas de la TCMD incluyen ser un examen no invasivo, la posibilidad de evaluación de las estructuras intratorácicas y que podría entregar una mejor evaluación de la BM. Por otra parte, sus desventajas incluyen la exposición a radiación, la necesidad de cooperación del paciente 0 de anestesia general, y la posibilidad de subestimar el grado de colapso en pacientes intubados o traqueostomizados ${ }^{20}$.

La TC también permite realizar técnicas de angiografía (AngioTC) que permiten la evaluación simultánea de la patología vascular, mediastínica y pulmonar ${ }^{8}$. En los últimos años, se han realizado broncoscopías virtuales con reconstrucciones 3D, obteniendo resultados variables ${ }^{21}$. Aunque la resonancia magnética (RM) permite una extensa evaluación anatómica, demora más tiempo, delinea de peor manera la vía aérea, muchas veces requiere anestesia general en niños, y es más cara. Por lo tanto, su uso se enfoca mayormente en la evaluación de TBM en pacientes con quistes, teratomas 0 alteraciones neurológicas, aunque en el futuro podría reemplazar a la AngioTC en la evaluación de TBM secundaria a alteraciones vasculares ${ }^{8}$.

\section{MANEJO}

Hasta ahora no existen guías de tratamiento basadas en evidencia y una revisión Cochrane de TM primaria no encontró evidencia de alta calidad para apoyar ninguna de las terapias usadas en la actualidad $^{22}$. Por lo tanto, la elección de la mejor estrategia depende principalmente de los síntomas 
y de la etiología, considerando también la tendencia a la resolución espontánea.

\section{Tratamiento no quirúrgico}

Para casos leves, una observación activa con kinesioterapia respiratoria, prevención y tratamiento del reflujo gastroesofágico y de las infecciones respiratorias recurrentes es una conducta adecuada y suficiente. Algunos casos moderados pueden ser manejados exitosamente con ventilación no invasiva con presión positiva (CPAP o BiPAP), que evita el colapso traqueal actuando como un stent neumático. A pesar de esto, en general se considera como una medida transitoria mientras el paciente se estabiliza, debido a la necesidad de soporte médico constante ${ }^{20}$. En esta línea, se publicó un caso de TM que logró ser manejado con una cánula nasal de alto flujo de manera ambulatoria, esperando la resolución espontánea de los síntomas ${ }^{23}$. Las desventajas pueden incluir retraso en la alimentación oral, habla/lenguaje y del desarrollo ${ }^{17}$. También, el uso de máscaras nasales de CPAP podría tener un potencial efecto negativo sobre el desarrollo medio-facial y dental a largo plazo ${ }^{24}$.

\section{Tratamiento quirúrgico}

Los síntomas severos que podrían indicar la necesidad de cirugía incluyen infecciones respiratorias recurrentes y graves, intolerancia al ejercicio, bronquiectasias y extubación fallida. La aparición de uno o más episodios de ALTE o "dying spells" debiera ser considerado como indicación de algún tratamiento quirúrgico ${ }^{1}$. Para estos casos existen distintas alternativas, Io que refleja que no existe un único tratamiento capaz de corregir todas las alteraciones y con poca morbilidad. La elección debe considerar la localización, extensión y etiología de la malacia. Si la condición clínica lo permite, se debe intentar evitar procedimientos muy invasivos o definitivos dada la tendencia de la TM (en especial la primaria) a mejorar con el tiempo.

\section{a. Traqueostomía}

Permite un bypass de la obstrucción en la zona malácica y también, en TM difusas, una traqueostomía baja con una cánula larga puede entregar soporte endoluminal, evitando el colaps $0^{20}$. Además, permite entregar apoyo ventilatorio y aspirar secreciones, aunque no corrige el problema primario y se asocia a todas las desventajas y complicaciones propias a las traqueostomías. Aun así, a veces es el único tratamiento posible debido a la inestabilidad del paciente 0 a la falla de otros tratamientos. Por otro lado, una traqueostomía bien indicada podría evitar la necesidad de cirugías complejas y riesgosas, en espera de la resolución espontánea.

\section{b. Resección y anastomosis (con o sin traqueoplastía)}

La resección del área comprometida de la tráquea malácica con anastomosis término-terminal 0 con una traqueoplastía por deslizamiento podría estar indicada en casos seleccionados de malacias muy localizadas. De ser posible, se aconseja no resecar más de $30 \%$ de la tráquea en pacientes pediátricos, debido a la menor resistencia a la tensión en la anastomosis ${ }^{6}$.

\section{c. Suspensión y/o reconstrucción traqueal transcervical}

En los casos de TM más proximales en la tráquea cervical y en los casos de malacia con colapso supraestomal en pacientes con traqueostomía previa, la suspensión cricotraqueal a los tejidos fasciomusculares o la reconstrucción con cartílago mediante abordajes cervicales pueden ser alternativas válidas ${ }^{25}$.

\section{d. Aortopexia}

Es una de las alternativas más utilizadas, en especial si la malacia es distal (como las que se asocian a atresia esofágica y fístula traqueoesofágica). La aorta se suspende del esternón, traccionando indirectamente la tráquea hacia anterior. El abordaje puede ser mediante esternotomía, toracotomía 0 toracoscopía ${ }^{26}$. La aortopexia limita el colapso traqueal anterior, aunque podría ser menos efectiva en el manejo del prolapso de la pared traqueal posterior membranosa ${ }^{1}$. La efectividad del procedimiento se considera $>80 \%$, aunque las complicaciones alcanzan $16,6 \%$ y la mortalidad $6 \%$ (muchas veces debido a las comorbilidades más que a la cirugía en sí) $)^{27}$. 


\section{e. Traqueopexias}

Recientemente se han descrito las técnicas de traqueopexia anterior y posterior, con buenos resultados a corto plazo. La primera fija la pared anterior de la tráquea al esternón, mientras que la segunda fija la membrana traqueal posterior al ligamento espinal longitudinal anterior ${ }^{28}$.

\section{f. Estabilización traqueal extraluminal}

La estabilización traqueal externa mediante el uso de tutores (splints) autólogos o protésicos permite entablillar la tráquea malácica. El riesgo de potenciales complicaciones como infección 0 erosión de estructuras adyacentes con el uso de materiales no-reabsorbibles ha volcado el interés hacia estabilizadores externos reabsorbibles, los que además permitirían el crecimiento de la vía aérea pediátrica. En los últimos años se ha reportado del uso de impresoras 3-D para fabricar moldes reabsorbibles a medida 29 .

\section{g. Stents intraluminales}

Son una idea muy atractiva debido a que pueden ser instalados de manera mínimamente invasiva mediante broncoscopía o radiología intervencional. A pesar de que existe una vasta experiencia en su uso en adultos para distintas indicaciones, su uso en pacientes pediátricos sigue siendo controvertido. El pequeño diámetro de la vía aérea; la posibilidad de migración, fractura u obstrucción; el riesgo de erosión hacia estructuras vecinas; la formación de tejido de granulación; la dificultad en su cambio o retiro; la necesidad de broncoscopías repetidas; y la necesidad de permitir el crecimiento de la vía aérea, son todos problemas a considerar respecto a su uso para el manejo de la TM pediátrica ${ }^{6,29}$.

Los cuatro tipos principales de stents de vía aérea son: de silicona, metálicos expansibles mediante balón, metálicos autoexpansibles, y bioabsorbibles. El stent ideal debiera ser de fácil

\section{BIBLIOGRAFÍA}

1. Fraga JC, Jennings RW, Kim PC. Pediatric tracheomalacia. Semin Pediatr Surg 2016; 25: 156-64

2. Pohunek P, Boogatard R, Merkus P. Congenital and Acquired Abnormalities of the Lower Airways. inserción y retiro, proveer una adecuada expansión de la vía aérea, ser bien tolerado por el organismo, no interferir con la función mucociliar, tener una adherencia segura a la pared traqueobronquial y estar disponible en una amplia gama de tamaños ${ }^{6,30}$. En la actualidad, ninguno cumple con todas estas características. Debido a sus complicaciones, el uso de stents en TM pediátrica se limita generalmente a uso paliativo, cuando otros tratamientos quirúrgicos han fallado, o para estabilizar la vía aérea después de una cirugía ${ }^{19,31}$.

\section{CONCLUSIÓN}

La TM es una patología que parece ser más frecuente que lo pensado previamente y puede generar síntomas respiratorios persistentes. Es fundamental tener un alto nivel de sospecha en pacientes pediátricos con síntomas de asma recurrente de difícil manejo y en pacientes con condiciones reconocidamente asociadas a TM, tales como atresia esofágica y malformaciones vasculares, para lograr un diagnóstico y tratamiento oportunos.

Existen múltiples alternativas terapéuticas y no existe evidencia suficiente para determinar cuál es la mejor estrategia, por lo que el tratamiento debe ser evaluado caso a caso. La gran mayoría de las TM primarias se resolverán antes de los 2 años, por lo que casos leves tienden a ser manejados de manera conservadora. En casos más sintomáticos, el apoyo ventilatorio no invasivo podría ser considerado como una herramienta más dentro del manejo de estos pacientes. Para pacientes con síntomas severos, existe una gran variedad de alternativas quirúrgicas y endoscópicas. Cada una tiene sus ventajas y desventajas, por lo que deben ser conocidas por los equipos encargados del cuidado de estos pacientes.

En: Priftis KN, Anthracopoulos MB, Eber E, Koumbourlis AC, Wood RE, eds. Paediatric Bronchoscopy. Basel, Switzerland: Karger, 2010; 130-40.

3. Walloo MP, Emery JL. The trachea in children with tracheo-oesophageal fistula. Histopathology 1979; 3: 329-38. 
4. Boogatid R, Huijsmans SH, Pijnenburg MW, Tiddens HA, de Jongste JC, Merkus PJ. Tracheomalacia and bronchomalacia in children: incidence and patient characteristics. Chest 2005; 128: 3391-7.

5. LeE EY, BoIseLLe PM. Tracheobronchomalacia in infants and children: multidetector CT evaluation. Radiology 2009; 252: 7-22.

6. Wallis C, McLaren CA. Tracheobronchial stenting for airway malacia. Paediatr Respir Rev2018; 27: 48-59.

7. Murgu S, Colt H. Tracheobronchomalacia and excessive dynamic airway collapse. Clin Chest Med 2013; 34: 527-55.

8. Deacon JWF, Widger J, Soma MA. Paediatric tracheomalacia - A review of clinical features and comparison of diagnostic imaging techniques. Int J Pediatr Otorhinolaryngol 2017; 98: 75-81.

9. Fayoux P, Morisse M, Sfeir R, Michaud L, Daniel S. Laryngotracheal anomalies associated with esophageal atresia: importance of early diagnosis. Eur Arch Otorhinolaryngol 2018; 275: 477-81.

10. Triglia JM, Nicollas R, Roman S. Tracheomalacia in Children. En: Graham JM, Scadding GK, Bull PD, eds. Pediatric ENT. Berlin Heidelberg, Germany: Springer-Verlag, 2007; 241-5.

11. Rogers DJ, Cunnane MB, Hartnick CJ. Vascular compression of the airway: establishing a functional diagnostic algorithm. JAMA Otolaryngol Head Neck Surg 2013; 139: 586-91.

12. Stojanovska J, Cascade PN, Chong S, Quint LE, SundaRAm B. Embryology and imaging review of aortic arch anomalies. J Thorac Imaging 2012; 27: 73-84.

13. McLaren CA, Elliott MJ, Roebuck DJ. Vascular compression of the airway in children. Paediatr Respir Rev 2008; 9: 85-94.

14. MonNier P. Congenital tracheal anomalies. En: Monnier P, ed. Pediatric Airway Surgery - Management of Laryngotracheal Stenosis in Infants and Children. Berlin Heidelberg, Germany: Springer-Verlag, 2011; 157-79.

15. Backer CL, Mongé MC, Popescu AR, Eltayeb OM, Rastatter JC, Rigsby CK. Vascular rings. Semin Pediatr Surg 2016; 25: 165-75.

16. Hysinger EB, Panitch HB. Paediatric Tracheomalacia. Paediatr Respir Rev 2016; 17: 9-15.
17. Carden Ka, Boiselle PM, Waltz DA, Ernst A. Tracheomalacia and tracheobronchomalacia in children and adults: an in-depth review. Chest 2005; 127: 984-1005.

18. Benjamin B, Cohen D, Glasson M. Tracheomalacia in association with congenital tracheoesophageal fistula. Surgery 1976; 79: 504-8.

19. Lee EY, Litmanovich D, Boiselle PM. Multidetector CT evaluation of tracheobronchomalacia. Radiol Clin North Am 2009; 47: 261-9.

20. Ngerncham M, Lee EY, Zurakowski D, Tracy DA, Jennings R. Tracheobronchomalacia in pediatric patients with esophageal atresia: comparison of diagnostic laryngoscopy/bronchoscopy and dynamic airway multidetector computed tomography. J Pediatr Surg 2015; 50: 402-7.

21. Su SC, Masters IB, Buntain H, et al. A comparison of virtual bronchoscopy versus flexible bronchoscopy in the diagnosis of tracheobronchomalacia in children. Pediatr Pulmonol 2017; 52: 480-6.

22. Goyal V, Masters IB, Chang AB. Interventions for primary (intrinsic) tracheomalacia in children. Cochrane Database Syst Rev 2012 0ct 17; 10: CD005304.

23. Vézina K, Laberge S, Nguyen TTD. Home highflow nasal cannula as a treatment for severe tracheomalacia: A pediatric case report. Pediatr Pulmonol 2017; 52: E43-E45.

24. Roberts SD, Kapadia H, Greenlee G, Chen ML. Midfacial and Dental Changes Associated with Nasal Positive Airway Pressure in Children with Obstructive Sleep Apnea and Craniofacial Conditions. J Clin Sleep Med 2016; 12: 469-75.

25. Antón-Pacheco Jl, Villafruela M, López M, García G, Luna C, Martínez A. Surgical management of severe suprastomal cricotracheal collapse complicating pediatric tracheostomy. Int $J$ Pediatr Otorhinolaryngol 2008; 72: 179-83.

26. Jennings RW, Hamilton TE, Smithers CJ, Ngerncham M, Feins N, Foker JE. Surgical approaches to aortopexy for severe tracheomalacia. J Pediatr Surg 2014; 49: 66-71.

27. Torre M, Carlucci M, Speggiorin S, Elliott MJ. Aortopexy for the treatment of tracheomalacia in children: review of the literature. Ital J Pediatr 2012; 38: 62.

28. Bairdain S, Smithers CJ, Hamilton TE, et al. Direct tracheobronchopexy to correct airway collapse 
due to severe tracheobronchomalacia: Shortterm outcomes in a series of 20 patients. $J$ Pediatr Surg 2015; 50: 972-7.

29. ShIEH HF, JenNIINGS RW. Three-dimensional printing of external airway splints for tracheomalacia. $J$ Thorac Dis 2017; 9: 414-6.
30. Sztanó B, Kiss G, Máral K, et al. Biodegradable airway stents in infants - Potential life-threatening pitfalls. Int J Pediatr Otorhinolaryngol 2016; 91: 86-9.

31. Eber E, Antón-Pacheco JL, de Blic J, et al. eRS statement: interventional bronchoscopy in children. Eur Respir J 2017; 50. pii: 1700901. 